\title{
Kommentar zur Beziehung der FMH zu den Weiterbildungsstätten für Assistenzärztinnen und -ärzte
}

\author{
A. Vollenweider*
}

\footnotetext{
* im Auftrag des Vorstandes der Chefärztegesellschaft des Kantons Zürich und mit Unterstützung der Delegiertenversammlung des VLSS vom 30. März 2005
}

Dr. med. A. Vollenweider Chirurgische Klinik Kreisspital Männedorf CH-8708 Männedorf
Es ist erfreulich, wie der Zentralvorstand der $\mathrm{FMH}$, der Einfachheit halber im weiteren «FMH» genannt, allergrösstes Gewicht darauf legt, eine hochstehende Weiterbildung zum Facharzttitel zu unterstützen. Sie hat konsequenterweise die zentrale Stellung der Weiterbildungsstätten (WBS) erkannt und legt grossen Wert auf deren gründliche Evaluation, unter anderem mit regelmässigen Assistentenbefragungen. Dadurch bekommen die WBS einen unschätzbaren Input für mögliche Verbesserungen und Optimierungen. Die Ärzteschaft wird durch entsprechende, regelmässige Publikationen in der Schweizerischen Ärztezeitung auf dem laufenden gehalten [1-3, 5, 7].

Auch der Einsatz der Leiterinnen und Leiter der WBS für dieses Thema ist bisher ausserordentlich gross gewesen. Sie haben an der Ausgestaltung der Fragebogen mitgearbeitet, haben in einer an sie gerichteten Umfrage mit einer Rücklaufquote von rund $80 \%$ überdurchschnittlich gewissenhaft diese Fragestellungen beantwortet [5] und haben in der gleichen Umfrage zu zwei Dritteln angegeben, aufgrund der Umfrageergebnisse Veränderungsprozesse einzuleiten.

Der Bericht über die 8. Umfrage im Jahre 2004 [7] unter den Assistenzärztinnen und Assistenzärzten über ihre WBS hat nun aber viele Leiter dieser WBS erstaunt. Mit Befremden mussten sie feststellen, dass zur Gesamtbeurteilung ein neuer Kennwert definiert wurde, der z. B. im Fach Chirurgie diese Wertung im Mittel aller Stätten nur noch knapp genügend beurteilt - die FMH aber taxiert diese Bewertung schönfärberisch als «insgesamt sehr positiv» [7].

Die genauere Analyse dieser Fakten wirft aber in der Tat die Frage nach Stichhaltigkeit dieser Umfrage auf: Die rein subjektive Befragung (z. B.: «die jetzige Arbeitssituation entspricht meinen Idealvorstellungen») wird durch die Auswerter ungenügend relativiert [3] und tel quel übernommen, um Empfehlungen an die Weiterbildner auszusprechen sowie ungenügende Beurteilungen an die entsprechenden Fachgesellschaften weiterzuleiten.
Dabei bedient sich die FMH sehr fraglicher Ansätze, die im weiteren etwas genauer beleuchtet werden sollen: Ein Grossteil der Problematik zeigt sich in der Chirurgie geradezu exemplarisch, weshalb in der Folge die Beispiele aus diesem Bereich genommen werden, mutatis mutandis aber auch für die anderen Fächer gelten. Es fliessen zum Beispiel Fragen wie «Besuchen Sie regelmässig Weiterbildungsveranstaltungen ausserhalb der regulären Arbeitszeit?» nicht in die Evaluation ein und sogenannte «Benchmarks» werden recht subjektiv gesetzt. So werden im Fach Chirurgie alle Stätten mit mehr als 10 Weiterbildungsstellen miteinander verglichen. Das heisst konkret, dass die Universitäts- und Zentrumsspitäler mit starker Ausrichtung auf Lehre, Forschung und hochspezialisierte Versorgung in die gleiche Gruppe wie die kleineren Schwerpunktspitäler genommen werden, welche als Auftrag die erweiterte Grundversorgung haben. Um beim chirurgischen Fach zu bleiben, ist bei diesen von zentraler Wichtigkeit, den Weiterzubildenden die operativen Fähigkeiten beizubringen, währenddem es im Laufe der Weiterbildung Aufgabe der Zentrumsspitäler sein soll, die Kandidaten auch auf die Wichtigkeit der evidenzbasierten Medizin hinzuweisen und sich im Umgang mit wissenschaftlichen Publikationen vertraut zu machen. Gerade die Evidence-based Medicine wird aber in der Gesamtbeurteilung durch die FMH doppelt gewichtet, so dass systembedingt die Schwerpunktspitäler schlecht abschneiden müssen.

Dieser Eindruck, dass die FMH ihre Analysen und Folgerungen zu den WBS sehr einseitig und nicht umfassend durchführe, wird unterstützt durch die verschiedenen, in der Schweizerischen Ärztezeitung diesbezüglich publizierten Artikel $[1-3,5,7]$ :

So kann ein Satz wie «eine Reduktion der Wochenarbeitszeit wird vor allem in den operativen Fächern mehr Freiraum für die Weiterbildung schaffen» [3] bei den Weiterbildnern nur auf schieres Unverständnis stossen, nachdem sie mehr und mehr Schwierigkeiten bekommen, 
den Auszubildenden in der limitierten Zeit zumindest noch die notwendigste operative Fertigkeit beizubringen [4].

An keiner Stelle wird durch die FMH betont, dass aus den Umfrageergebnissen eine erschreckende Passivität und Konsumhaltung der Auszubildenden zuTage tritt (nur 43\% der Assistentinnen und Assistenten lesen das Weiterbildungskonzept ihrer WBS, weniger als die Hälfte benützen Wartezeiten oder Pausen regelmässig für die eigene Weiterbildung; Umfrage 2004). Nirgends wird in den Kommentaren auch nur angedeutet, dass in keinem akademischen Fach die Weiterbildung (Theorie und Praxis zusammen) mit einer 50-Stunden-Woche zu bewerkstelligen sei. Und auch die Tatsache, dass Assistentinnen und Assistenten, die in der Chirurgie ein Fremdjahr absolvieren, diese Weiterbildungsstätte signifikant schlechter beurteilen, führt lediglich zur Empfehlung der FMH, dass diese Stätten ihr Konzept verbessern sollen [3, 7]. Die Verantwortlichen vor Ort nehmen aber sehr häufig wahr, dass gerade im chirurgischen Fach überdurchschnittlich häufig direkte Staatsexamenabgänger ihre Weiterbildung erst beginnen. Und diese erstmalige Konfrontation mit der klinischen Verantwortung ist für die jungen Assistentinnen und Assistenten sehr belastend und oft mit negativen Gefühlen verbunden, was letztlich wohl unumgänglich ist und von verantwortungsvollen Ausbildern auch gefordert werden muss.

Zusammenfassend erscheint es dringend notwendig, dass die FMH ihre Haltung gegenüber den Leitern der Weiterbildungsstätten differenziert und diesen vermehrt Verständnis entgegenbringt. Sie sind nämlich, wie die Umfrage [5] zeigt, hochmotiviert, diese zentrale Ausbidungsfunktion weiterhin nach bestem Wissen und Gewissen zu erfüllen. Sie stehen aber wie so viele Exponenten im Gesundheitswesen unter zunehmendem Druck auch von Seiten der Qualitätsund Controllinganforderungen, der Arbeitszeit- vorschriften und nicht zuletzt des immer engeren Kostendaches - die Äusserung von jedem 2. Leiter einer WBS, dass mit Spitalärzten bei mindestens gleicher Qualität kostengünstiger gearbeitet werden könnte [5], müsste eigentlich ein Alarmzeichen sein für die Weiterbildungsverantwortlichen bei der FMH!

Aus Sicht der verantwortlichen Weiterbildner erweist die FMH der zukünftigen Ärztegeneration einen Bärendienst, wenn sie zu einseitig die Weiterbildungsstätten unter Druck setzt. Viel eher müsste den Auszubildenden auch durch die FMH klargemacht werden, dass der Arztberuf wegen seiner Wichtigkeit für die Gesellschaft nach wie vor zu Recht ein hohes Sozialprestige aufweist [6], dieses aber auch ein überdurchschnittliches Engagement erfordert. Entsprechend ist für diesen Beruf ohne vermehrte Selbstinitiative keine Weiterbildung möglich. Ein erneuter intensiver Dialog zu diesem Thema ist daher dringend nötig.

\section{Literatur}

1 Giger M, Siegrist M. Ein neuer Fragebogen zur Beurteilung der Weiterbildungsstätten - was soll das? Schweiz Ärztezeitung 2003;84(31):1612-3.

2 Giger M, Siegrist M. Ärztliche Weiterbildung auf dem Prüfstand. Schweiz Ärztezeitung 2003; 84(50):2655-7.

3 Siegrist M, Gutscher H, Giger M. Was haben Betriebskultur, Motivation und Fremdjahr mit der Weiterbildung zu tun? Schweiz Ärztezeitung 2004;85(15):783-91.

4 Schwöbel MG. Sind 50-Stunden-Woche und zeitgerechte Weiterbildung miteinander vereinbar? Schweiz Ärztezeitung 2004;85(34):1733-8.

5 Siegrist M, Giger M. Assistenten- und Assistentinnenumfrage 2003 aus Sicht der Leiter und Leiterinnen von Weiterbildungsstätten. Schweiz Ärztezeitung 2004;85(42):2262-6.

6 Univox-Trendbericht Bildung 2002/3. gfs-Zürich.

7 Siegrist M, Orlow P, Giger M. Weiterbildung aus der Sicht der Assistenzärzte. Schweiz Ärztezeitung 2005;86(7):412-23. 